\title{
Dem Mitarbeiter zu Diensten. Weiterbildung und Qualifizierung als Personennahe Dienstleistung
}

\author{
Gergana Vladova $(\mathbb{D} \cdot$ Alexander Heuts $\cdot$ Malte Teichmann
}

Eingegangen: 17. Januar 2020 / Angenommen: 16. Mai 2020 / Online publiziert: 5. Juni 2020

(C) Der/die Autor(en) 2020, korrigierte Publikation 2021

Zusammenfassung Die Weiterbildung und Qualifizierung der Mitarbeiter sind zentrale Erfolgsfaktoren des digitalen Wandels. Die zentrale Herausforderung besteht darin, diese maßgeschnitten anzubieten sowie notwendige Akzeptanz nicht vorauszusetzen, sondern ebenso als Zielgröße anzusehen. Dies geschieht jedoch nur, wenn die Mitarbeiter als Partner gesehen werden, deren Bedürfnisse und Verständnis nachhaltig berücksichtigt werden. Dieser Beitrag schlägt vor diesem Hintergrund einen Ansatz vor, Weiterbildung als Personennahe Dienstleistung zu realisieren. Dafür wird zuerst ein skizzenhafter Überblick über grundlegende Kompetenzanforderungen des digitalen Wandels gegeben. Danach wird die aktuelle Situation betrieblicher Weiterbildung in der digitalen Transformation beleuchtet. Hierzu wurde in einem Zeitraum von sechs Monaten im Rahmen einer quantitativen Untersuchung erhoben, wie Beschäftigte die digitale Transformation ihres Unternehmens und daraus resultierende Bedarfe betrieblicher Weiterbildung wahrnehmen. Darauf basierend werden drei aktuelle Paradoxe abgeleitet, die mit einer Durchführung von Weiterbildung als Personennahe Dienstleistung verhindert werden können. Empfehlungen

G. Vladova $\cdot$ M. Teichmann

Lehrstuhl für Wirtschaftsinformatik, insb. Prozesse und Systeme, Universität Potsdam,

Karl-Marx-Straße 67, 14482 Potsdam, Deutschland

E-Mail: malte.teichmann@wi.uni-potsdam.de

G. Vladova $(\bowtie) \cdot$ A. Heuts $\cdot$ M. Teichmann

Forschungsgruppe „Bildung und Weiterbildung in der digitalen Gesellschaft“, Weizenbaum-Institut für die vernetzte Gesellschaft, Hardenbergstraße 32, 10623 Berlin, Deutschland

E-Mail: gergana.vladova@wi.uni-potsdam.de

\section{A. Heuts}

E-Mail: alexander.heuts@hu-berlin.de

\section{A. Heuts}

Forschungsgruppe „Didaktik der Informatik / Informatik und Gesellschaft“, Humboldt-Universität zu Berlin, Unter den Linden 6, 10099 Berlin, Deutschland 
und Lösungsansätze werden hierzu diskutiert und weiterer Forschungsbedarf abgeleitet.

Schlüsselwörter Digitale Transformation · Kompetenzentwicklung ·

Weiterbildung · Industrie $4.0 \cdot$ Personalisierung $\cdot$ Personennahe Dienstleistungen

\title{
At the Service of the Employee. Further Training and Qualification as a Personal Service
}

\begin{abstract}
The further training and qualification of employees are central success factors of digital change. The central challenge is to offer these customized services and not to presuppose acceptance, but rather to regard it as a target value. However, this will only happen if the employees are seen as partners and their needs and understanding are taken into account in the long term. Against this background, this article proposes an approach to realize further education as a personal service. For this purpose, a brief outline of the basic competence requirements of digital change is given first. Afterwards, the current situation of in-company continuing training in the digital transformation will be examined. A quantitative survey was conducted over a period of six months to determine how employees perceive the digital transformation of their company and the resulting needs for continuing vocational training. Based on this, three current paradoxes are derived, which can be prevented by conducting continuing education as personal service. Recommendations and solutions will be discussed and further research is needed.
\end{abstract}

Keywords Digital transformation - Competence development · Further education . Industry $4.0 \cdot$ Personalization $\cdot$ Personal service

\section{Digitale Transformation und erweiterte Kompetenzanforderungen}

Eine Definition der Digitalisierung aus dem Jahr 1998 beschreibt diese als „encoding information as a stream of bits" (Shapiro et al. 1998) - als die Umwandlung aller Arten von Informationen und Medien - Text, Ton, Fotos, Videos, Daten - von Instrumenten und Sensoren in Einsen und Nullen - die natürliche Sprache der Maschine. Wird heute jedoch über Digitalisierung gesprochen, werden viele Bereiche und Themen, z. B. Lernen und Qualifizierung, neue Geschäftsmodelle, neue Staatsbürgerschaft und Rechtsnormen adressiert. Die zum jetzigen Zeitpunkt stattfindenden Entwicklungen weisen hinsichtlich des Umfangs und der Geschwindigkeit ein besonderes Momentum in der menschlichen Kulturgeschichte auf. Nur ansatzweise sind mögliche Folgeerscheinungen auf gesellschaftliche Zusammenhänge benennbar, welche über Chancen revolutionärer digitaler Partizipationsformate bis hin zu Risiken einer möglichen „Erosion der Demokratie“ (Zeit 2017) reichen.

Unter dem Stichwort „Industrie 4.0“ werden auch in Unternehmen fortlaufend technische, organisatorische und institutionelle Wandlungsprozesse angestoßen. Mit dem fortschreitenden digitalen Wandel entsteht ein neues soziotechnisches System, in welchem die Anforderungen an die Qualifikationen passend definiert und die 
Qualifizierungs- und Weiterbildungsangebote unter Berücksichtigung der veränderten Rahmenbedingungen und Anforderungen gestaltet werden müssen (Kagermann et al. 2013). Dies betrifft alle Mitarbeitergruppen - gut ausgebildete multidisziplinäre Spezialisten, die die Entwicklung und Einführung der neuen Technologien organisieren und leiten als auch die Beschäftigten mit geringen Qualifikationen, deren Handlungsmöglichkeiten eventuell beeinträchtigt werden (Russwurm 2015; Hirsch-Kreinsen 2014). Konsens herrscht darüber, dass diese Anforderungen nur durch eine hohe Lern- und Innovationsfähigkeit der Beschäftigten adressierbar sind. Dazu zählt die Fähigkeit, Herausforderungen im Arbeitsalltag (kritisch) einschätzen und analysieren (Calvani et al. 2009) sowie diese mithilfe digitaler Technologien adressieren zu können. Ebenfalls sind ein hohes Maß an Kreativität sowie eine ausgeprägte Innovationsbereitschaft (u. a. Battelle for Kids 2019; Ramos et al. 2018; Hermann et al. 2017) unabdingbar, um Lösungsansätze zu generieren und umzusetzen. Die Zunahme wissensintensiver Tätigkeiten bei gleichzeitiger Abnahme an- und ungelernter Tätigkeiten ist unter dem Begriff ,,kkill-biased technical change“ subsumierbar (z. B. Brynjolfsson und McAfee 2016; Acemoglu und David 2011) und ein branchenübergreifendes Phänomen. Im Bereich unternehmensbezogener Dienstleistungen beziffern z. B. Dengler und Matthes (2015) das technikinduzierte Substituierungspotenzial von Hilfstätigkeiten mit $40 \%$.

Bei allen Vorteilen, die dem digitalen Wandel zugeschrieben werden, sind Unterschiede beim Akzeptanzverhalten der Mitarbeiter zu beobachten (Klenow 1998; Rai 1996). Der Prozess der digitalen Transformation sollte vor diesem Hintergrund nicht allein einem Top-down-Ansatz folgen. Ebenso sind Bottom-up orientierte Zugänge zu berücksichtigen, da diese als Wandlungs- und Innovationstreiber dienen (Vladova et al. 2017). Aus dem unmittelbaren Umgang mit Technologien, Werkzeugen oder Methoden entstehen nicht selten Ideen zur Weiterentwicklung von Mitarbeitern, da diese Bedarfe an akuten Prozessverbesserungen erkennen. Beteiligung wird damit zum wesentlichen Faktor der Akzeptanzsteigerung (ebd.).

Der Mensch bleibt branchenübergreifend ein kritischer Erfolgsfaktor der Digitalisierung, dessen Potenziale jedoch auf Basis individueller Dispositionen - Kenntnisse, Kompetenzen und Akzeptanz - entwickelt werden müssen. Ansätze zur Betrachtung von Weiterbildung als personennahe Dienstleistung (PD) knüpfen an den erkannten Bedarf passender und individualisierter Weiterbildungsangebote an. Insbesondere die Identifikation mitarbeiterindividueller Kompetenzentwicklungsbedarfe stellt dabei eine Herausforderung dar. Wissen über die Vorkenntnisse der Beschäftigten ist dafür essentiell (Paproth und Kittel 2019). Darüber hinaus erfordert der digitale Wandel die fachliche Entwicklung von Fachkräften bzw. die Nachqualifizierung An- und Ungelernter. In der Vergangenheit war diesbezüglich jedoch Verbesserungspotenzial erkennbar: Beispielhaft boten 2016 nur 23,1\% der Unternehmen explizit Industrie 4.0-adressierte Weiterbildungen an (acatech 2016).

Vor diesem Hintergrund bieten die nachfolgend vorgestellten Ergebnisse der empirischen Untersuchung einen Überblick, wie Beschäftigte die aktuelle Weiterbildungssituation in der digitalen Transformation einschätzen. Es liegt der Fokus auf der Frage, inwieweit die Weiterbildung zurzeit als individualisiert und personennah gelebt und wahrgenommen wird. 


\section{Untersuchungsverlauf und -rahmen}

Für die Erhebung wurde ein quantitativer Ansatz mit qualitativen Elementen gewählt. Mithilfe eines Fragebogens wurden die Teilnehmenden unter anderem zu verschiedenen Aspekten des digitalen Wandels, zum allgemeinen Weiterbildungsangebot in den Unternehmen, zum digitalen Weiterbildungsangebot im Speziellen und zu den Mitarbeiterkompetenzen befragt. Im Anschluss sollten die Befragten noch eine Prognose bezüglich des zukünftigen Bedarfs und der Erwartungen an Weiterbildungsangebote geben.

Die Umfrage wurde im Zeitraum von April 2019 bis Oktober 2019 durchgeführt. Insgesamt wurden 171 Mitarbeitende deutscher und international agierender Unternehmen befragt. Die Teilnehmenden wurden direkt angesprochen. Ein kleinerer Teil wurde über die Plattform Amazon Mechanical Turk akquiriert. In diesem halben Jahr wurde die Befragung mit der Ausgangssprache Deutsch zu rund 72\% aufgerufen. Die restlichen Fragebögen wurden auf Englisch ausgefüllt. In der Umfrage sind Mitarbeiter in Unternehmen unterschiedlicher Größen vertreten. Die Datensammlung umfasst nach einer Bereinigung insgesamt 103 Einträge.

Im Fragebogen wurde zur Ermittlung von persönlichen Einstellungen oder $\mathrm{Zu}$ stimmungen eine 4-stufige Likert-Skala verwendet, wobei Antwortmöglichkeiten von ,stimme voll und ganz zu“ bis ,stimme gar nicht zu“ verwendet wurden. Optional stand jeweils noch eine fünfte Antwortmöglichkeit zur Verfügung, falls eine befragte Person eine Aussage nicht beurteilen konnte. Für diese Art von Fragen ergibt sich somit eine Ordinalskala. Auch bei Fragen zur Nennung von bestimmten Inhalten, Möglichkeiten, Problemen, Angeboten, etc. wurden die Antwortmöglichkeiten vorgegeben. Falls keine der Antworten oder weitere nicht aufgelistete Möglichkeiten zur Beantwortung einer Frage relevant waren, so gab es in den meisten Fällen für die Teilnehmenden eine zusätzliche Auswahl mit der Möglichkeit einer freien Antwort. Bei diesen Fragen liegt eine Nominalskala vor. Weiterhin wurde bei einem Großteil der Fragen auch die Möglichkeit geboten, die gewählten Antworten zu kommentieren, um zusätzliche Informationen zur Auswahl zu erhalten.

Knapp ein Drittel der befragten Personen $(n=103)$ gab an, dass in ihrem Unternehmen mehr als 1000 Mitarbeitende tätig sind. Jeweils rund 16\% der Befragten gaben an, dass bei ihnen 5 bis 50, 51 bis 150 und 501 bis 1000 Personen beschäftigt sind. Zwischen fünf und neun Befragte teilten mit, dass in ihren Unternehmen weniger als 5, 151 bis 250 und 251 bis 500 Personen arbeiten. Die Teilnehmenden sind darüber hinaus in den verschiedensten Branchen tätig, die Umfrageergebnisse sind somit nicht für eine bestimmte Branche repräsentativ. Mit rund $15 \%$ waren in der Elektrotechnik tätige Personen am meisten vertreten. Die Hälfte aller Befragten befindet sich in der Altersgruppe von 20 bis 30 Jahren. Rund ein Viertel war zwischen 31 und 40 Jahren alt. In etwa $10 \%$ der befragten Personen gab an, dass sie zwischen 41 und 50 Jahren alt sind. Knapp 14\% gaben an, dass sie 51 Jahre und älter sind. $45 \%$ der Befragten arbeiten weniger als 5 Jahre in ihrem Betrieb. Bei rund einem Drittel liegt die Betriebszugehörigkeit bei 5 bis 10 Jahren und bei $6 \%$ bei mehr als 25 Jahren. Zum Geschlecht oder zur Ausbildung wurden keine Daten erhoben. 


\section{Zentrale Untersuchungsergebnisse}

Nachfolgend werden die zentralen Ergebnisse der Untersuchung vorgestellt. Zuerst wird gezeigt, wie Beschäftigte den Umgang Ihres Unternehmens mit dem digitalen Wandel bzw. damit verbundener Weiterbildungsaktivitäten einschätzen. Daran anschließend werden Entwicklungspotenziale für die Weiterbildung im digitalen Wandel aus Sicht der Beschäftigten aufgezeigt.

\subsection{Weiterbildung im Kontext des digitalen Wandels}

Rund drei Viertel der Befragten stimmten voll und ganz oder größtenteils der Aussage zu, dass sich ihre Organisation aktiv mit Industrie 4.0-induzierten Veränderungen auseinandersetzt. Ebenfalls ungefähr drei Viertel aller Befragten gaben an, dass durch den digitalen Wandel neue Technologien eingeführt und angewendet werden. In etwa der Hälfte aller Fälle hat der digitale Wandel bereits Prozesse und Abläufe über alle Organisationsebenen verändert. Die abteilungsübergreifende Organisation hat sich in den Unternehmen zu knapp $57 \%$ verändert. Weiterhin teilten in etwa $40 \%$ der befragten Personen mit, dass sich konkrete Aufgaben und Tätigkeiten in der Produktion verändert haben. Auf der Ebene der betrieblichen Weiterbildung findet die Transformation allerdings nicht so intensiv statt - aus Sicht der Beschäftigten ist das in rund $40 \%$ der betreffenden Unternehmen der Fall.

\subsubsection{Strategische Ebene betrieblicher Weiterbildung}

Als Herausforderungen für die Weiterbildung zur Umsetzung von Industrie 4.0 werden von mehr als der Hälfte der Befragten sowohl fehlendes Fachwissen der Beschäftigten als auch Kompatibilitätsprobleme zwischen neuen und alten Prozessen oder Technologien identifiziert. Etwas weniger als die Hälfte gab zu bedenken, dass ein mangelnder Reifegrad der Technologien im Unternehmen eine Herausforderung bei der Umsetzung ist. Etwa zwei von fünf Befragten gaben an, dass eine fehlende bzw. eine geringe Beteiligung der Beschäftigten bei Diskurs und Umsetzung von Industrie 4.0-orientierter Weiterbildung eines der größten Probleme ist. $\mathrm{Zu}$ wenig Weiterbildungsangebote zu diesen Themen sehen rund $37 \%$ der Personen als problematisch. Rund 30\% stufen unzureichende Kenntnisse über Datensicherheit und Industrie 4.0 bei den Verantwortlichen oder den Beschäftigten als eine große Herausforderung ein. Knapp $28 \%$ teilten mit, dass unzureichende Kenntnisse über den aktuellen Forschungsstand zum Thema bei den Verantwortlichen hemmend in der Umsetzung wirken. Lediglich rund ein Viertel gab an, dass Umsetzungsprobleme durch ausufernde Kosten verursacht werden.

Mehr als $70 \%$ der befragten Personen gaben ebenfalls an, dass in ihrem Betrieb keine systematische Kompetenzerfassung durchgeführt wird. Bei $62 \%$ der Unternehmen ohne Erfassungssystem werden die Kompetenzen sogar in keiner Weise erfasst. In einigen Fällen erfolgt die Kompetenzfeststellung vereinzelt in Gesprächen mit den jeweils verantwortlichen Supervisoren. Die große Mehrheit gab gleichzeitig mit rund $90 \%$ an, dass die individualisierte Erfassung von Mitarbeiterkompetenzen wichtig sei, um daraus bspw. mögliche Weiterbildungsbedarfe abzuleiten. Weiterhin 
stimmten mehr als drei Viertel der Befragten der Aussage zu, dass die Anforderungen an die Mitarbeiter durch den digitalen Wandel schneller steigen würden als notwendige Kompetenzen real entwickelt werden könnten.

Ungefähr $75 \%$ aller befragten Mitarbeitenden gaben weiterhin an, dass ihr Unternehmen digitale Lernformate anbietet. In diesem Zusammenhang stimmen etwa zwei Drittel aller teilnehmenden Personen voll und ganz bzw. größtenteils der Aussage zu, dass sie digitale Weiterbildungsangebote genauso gern oder sogar lieber als herkömmliche, analoge Angebote nutzen. Knapp zwei Drittel der Befragten gaben an, das Gefühl zu haben, dass sie in ihrem Unternehmen gut auf die Veränderungen vorbereitet werden und ausreichende Informationen erhalten, was die Digitalisierung für sie als Mitarbeitende bedeutet.

\subsubsection{Operative Ebene betrieblicher Weiterbildung}

Die Unternehmen stellen ihren Beschäftigten diverse Lernmöglichkeiten zur Verfügung. Über $60 \%$ der befragten Personen gaben an, dass ihnen internetbasierte Angebote wie z. B. Foren, Webseiten oder Lernplattformen angeboten werden. Häufig informieren und bilden sich Beschäftigte jedoch auch durch Gespräche mit Kollegen bzw. deren Hilfe oder durch reines Beobachten und Ausprobieren fort. In rund $38 \%$ der Unternehmen werden weiterhin digitale Lernprogramme angeboten. $\mathrm{Zu}$ den gängigsten Angeboten gehören interne Weiterbildungsangebote in

Internetbasierte Angebote (z. B. Foren, Webseiten, Lernplattformen)

Gespräche/Hilfe von Kollegen

Interne Weiterbildungsangebote im Unternehmen in Form von Präsenzveranstaltungen durch Externe

Beobachten/Ausprobieren

Digitale Lernprogramme

Offizielle Weiterbildungen durch andere Mitarbeitende

Informationsveranstaltungen

Fachbücher/Fachzeitschriften

Besuch von Messen

Externe Weiterbildungsangebote im Unternehmen in Form von Präsenzveranstaltungen

Learning on the Job-Angebote

Diskussionveranstaltungen (analog)

Informationsbroschüren

Simulationen

Massive Open Online Courses (MOOC)

0

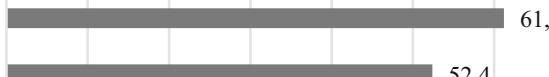

61,2

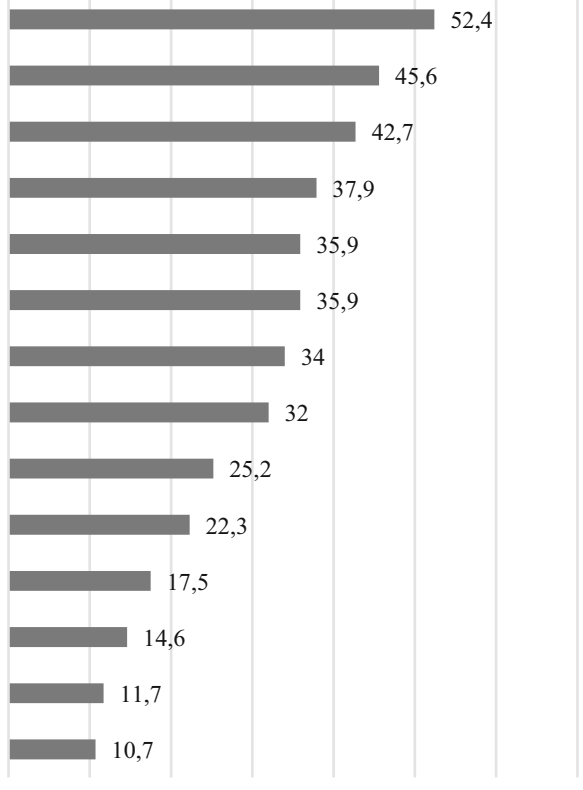

Zustimmung der Aussage in \%

Abb. 1 Möglichkeiten seitens der Unternehmen, um Mitarbeitende zu informieren und weiterzubilden 
Form von Präsenzveranstaltungen durch Externe, Informationsveranstaltungen oder der Besuch von Messen. Massive Open Online Courses (MOOC) und Simulationen werden hingegen eher selten angeboten (siehe Abb. 1).

Der Zeitpunkt von Weiterbildungen wird von ungefähr einem Viertel der Personen als unpassend empfunden. Gründe dafür sind unter anderem, dass dafür teilweise freie Tage geopfert werden müssen, die Zeiträume im Widerspruch zu den täglichen Aufgaben stehen und dadurch eine Teilnahme nicht möglich ist. Von den befragten Personen gab jede fünfte an, dass die Dauer der Weiterbildungsangebote unpassend (zu kurz oder zu lang) ist. Genannte Gründe dafür sind etwa zeitliche Konflikte mit der eigentlichen Arbeit oder dass Angebotszeiten als zu unflexibel eingestuft wurden bzw. Maßnahmen oft abends stattfinden. Ebenfalls gab jede fünfte Person an, dass der Inhalt der Weiterbildung keine Relevanz für sie hat. Oft wurden Inhalte als wenig spannend, teilweise zu generisch oder auch nicht für die eigentliche Arbeit relevant kritisiert. Die Gestaltung der Weiterbildung stufte hingegen nur rund $15 \%$ der befragten Personen als uninteressant ein. Weiterhin empfanden die Befragten die Angebote, an denen sie bisher teilgenommen haben, zu $45 \%$ als häufig und lediglich zu $18 \%$ als immer sinnvoll. $9 \%$ der Befragten empfand diese als überhaupt nicht sinnvoll. Auch hier kristallisierte sich heraus, dass es zum Thema Digitalisierung oder Industrie $4.0 \mathrm{zu}$ wenig Angebote gibt bzw. verschiedenste neue Technologien oft fehlen oder zu spät adaptiert werden würden.

\subsection{Entwicklungspotenziale aus Sicht der Beschäftigten}

Um herauszufinden, womit sich die betriebliche Weiterbildung in Zukunft thematisch beschäftigen sollte, wurden die Befragten gebeten anzugeben, welche Inhalte ihnen derzeit fehlen. Mehr als die Hälfte gab an, dass tiefergehende Erklärungen zu veränderten Abläufen im Arbeitsalltag durch die Digitalisierung fehlen. Für knapp $45 \%$ gibt es Bedarf an technischen Schulungen in Bezug auf den Umgang mit

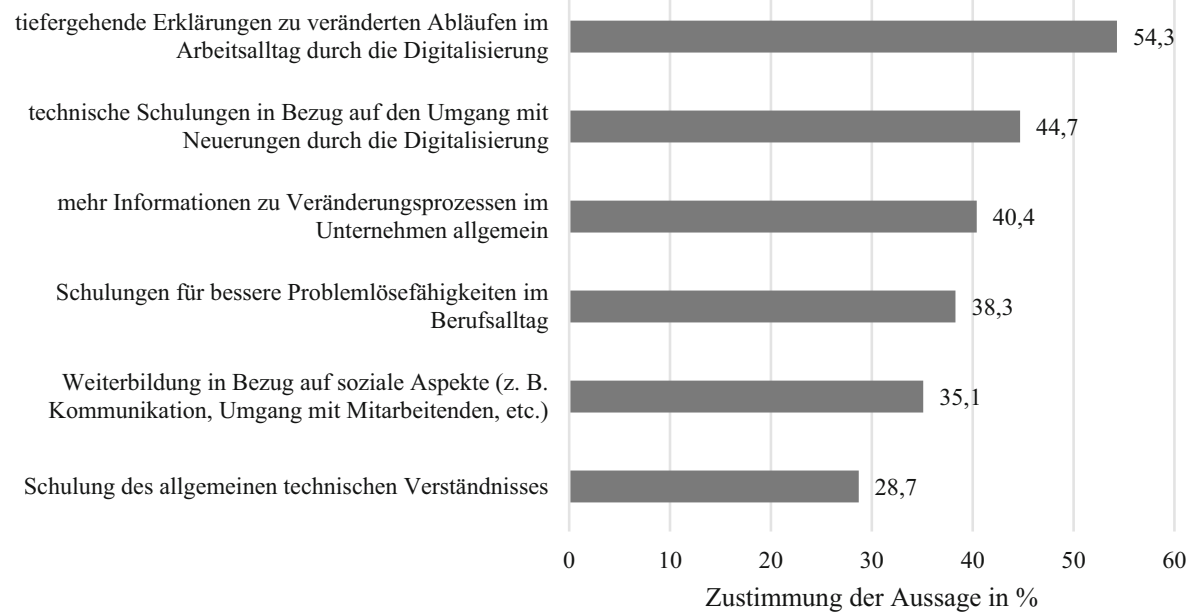

Abb. 2 Gewünschte Inhalte und Themen in der betrieblichen Weiterbildung 


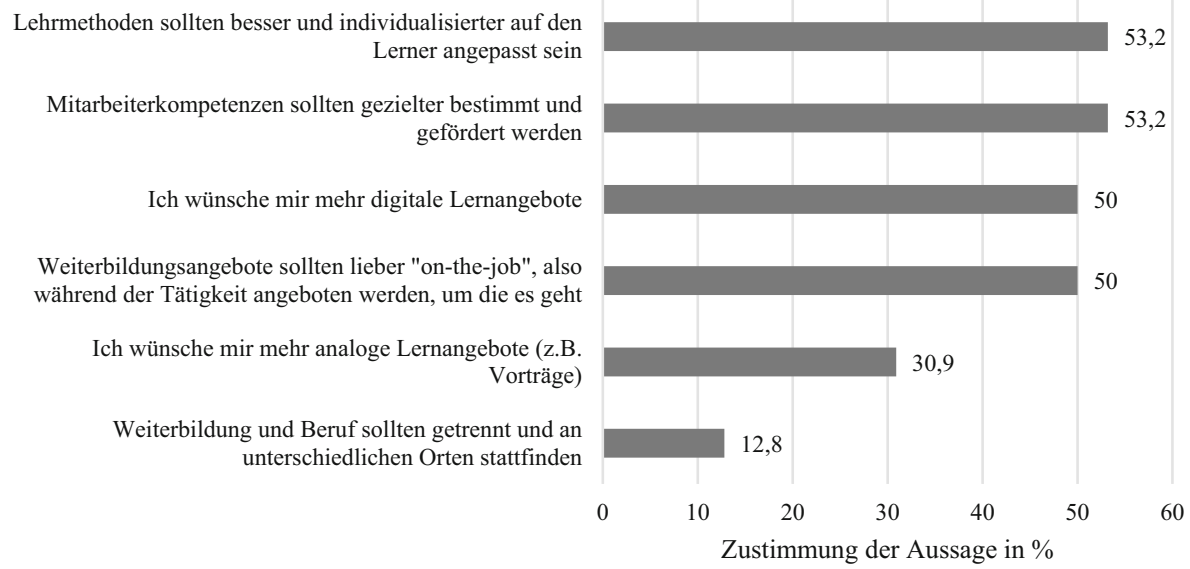

Abb. 3 Gewünschte Weiterbildungsmethoden in der Zukunft

Neuerungen durch die Digitalisierung. Jeweils in etwa 2 von 5 Personen vermissen Schulungen, die Kompetenzen für bessere Problemlösefähigkeiten im Berufsalltag bzw. Informationen zu Veränderungsprozessen im Unternehmen allgemein vermitteln. Bei rund 35\% der Befragten besteht Bedarf an Weiterbildung bezüglich Softskills, wie etwa Methoden zur Kommunikation und Umgang mit anderen Beschäftigten. Schlussendlich gaben rund $29 \%$ an, dass ihnen die Schulung des allgemeinen technischen Verständnisses fehlt (siehe Abb. 2).

Weiterführend wurde ebenfalls nach den Wünschen zu Formen zukünftiger Weiterbildung gefragt. Grundsätzlich besteht bei $54 \%$ der Befragten ein hoher Wunsch nach Individualisierung von Lehrmethoden. Hervorzuheben ist ebenfalls, dass sich mehr als die Hälfte der befragten Personen eine gezielte Bestimmung und Förderung von Mitarbeiterkompetenzen wünschen. Hier scheint eine hohe Sensibilität gegenüber der fehlenden systematischen Erhebung von Mitarbeiterkompetenzen vorzuliegen. Etwas mehr als die Hälfte wünscht sich mehr digitale Lernangebote, wohingegen knapp ein Drittel der Beschäftigten mehr analoge Angebote fordert. Die Hälfte aller Befragten würde ebenfalls ein verstärktes Angebot von Weiterbildungsformaten bevorzugen, die „on-the-job“, also während der Tätigkeit, realisiert werden können. Lediglich knapp ein Achtel aller Befragten würde eine Trennung von Weiterbildungsort und Berufsort bevorzugen. Mehrheitlich existiert folglich der Wunsch, berufliche Tätigkeit und Weiterbildung am selben Ort durchzuführen und damit zu verknüpfen (siehe Abb. 3).

\section{Betriebliche Weiterbildung als Personennahe Dienstleitung}

Für gegenwärtige Weiterbildung lassen sich vor dem Hintergrund der Untersuchungsergebnisse drei Paradoxe ableiten, die mit einer Durchführung von Weiterbildung als Personennahe Dienstleistung (PD) verhindert werden können. 
Paradox 1 Beschäftigte als Gruppe im Fokus, das Individuum jedoch nicht

Resümierend zeigt sich, dass thematisch allgemein ausgerichtete Weiterbildungsangebote vom Großteil der Unternehmen angeboten werden. Eine wesentliche Erkenntnis der Untersuchung ist jedoch auch, dass in den meisten Unternehmen kein systematisiertes Kompetenzerfassungssystem existiert, obwohl die Mehrheit der Beschäftigten die individualisierte Erfassung als wichtig einstuft. Das Paradox besteht folglich darin, dass Unternehmen gleichzeitig der Auffassung sein können, ausreichend Weiterbildung anzubieten, diese jedoch $\mathrm{zu}$ allgemein halten, sodass die eigentlich intendierten Effekte ausbleiben.

Wird Weiterbildung als PD realisiert, kann dem wichtigen Anspruch an eine systematische Kompetenzerfassung auf individueller Ebene Rechnung getragen werden. Werden individuelle Dispositionen wie Vorwissen, Kompetenzen und auch Akzeptanz in die Planung und Durchführung von Weiterbildung inkorporiert, können Bedarfe zielorientiert adressiert werden. Daran anschließend verringert sich ebenfalls die Gefahr, Kompetenzentwicklung an diesen konkreten Bedarfen vorbei zu realisieren. Durch die genaue Kenntnis über die Ausgangslage der Adressaten können notwendige Kompetenzen vielmehr identifiziert und zielorientiert entwickelt werden. Durch den Wegfall redundanter Entwicklungsprozesse wird es ebenfalls möglich, die Geschwindigkeit von Weiterbildung durch Individualisierung und Personalisierung an die des digitalen Wandels anzupassen.

Paradox 2 Die Digitalisierung öffnet Türen, die betriebliche Weiterbildung hat aber noch nicht den passenden Schlüssel parat

Technologien, die abteilungsübergreifende Organisation sowie unternehmensweite Prozesse werden aktuell als am meisten durch die Digitalisierung betroffen eingestuft. Betriebliche Weiterbildung hat sich dagegen nur sehr moderat verändert: Speziell auf das Thema Digitalisierung und Industrie 4.0 angepasste Fort- und Weiterbildungsangebote sind nur selten in ausreichenden Maßen vorhanden. Genau dieser thematische Fokus ist jedoch von Relevanz, um die Mitarbeiter auf den digitalen Wandel vorzubereiten und damit verbundene Potenziale zu heben. Statt eines allgemeinen technischen Verständnisses sind vielmehr tiefergehende Erklärungen zu veränderten Abläufen im Arbeitsalltag sowie der Umgang mit digitalen Technologien in Wertschöpfungsprozessen zu adressieren. Konventionelle Anpassungsschulungen, die primär auf die kontextlose Bedienung einzelner Technologien abzielen, greifen damit zu kurz. Kritisch ist, dass diese im Vergleich zu themenspezifischen Industrie 4.0-verbundenen Weiterbildungsangeboten übermäßig angeboten werden.

Wie bereits dargelegt, hat Weiterbildung als PD realisiert das Potenzial, zielgerichteten Kompetenzerwerb durch Identifizierung von Kompetenzbedarfen zu ermöglichen. Ebenfalls wächst durch das daraus resultierende in den Fokus setzen der Mitarbeiter bzw. derer Bedürfnisse die Chance, dass auch von der operativen Durchführung entkoppelte Entscheidungsträger (z. B. Organisatoren von Weiterbildung oder Human Ressources) ein tiefergehendes Verständnis von den Auswirkungen des digitalen Wandels bekommen. Eine Organisation von Weiterbildung, die bestehende fachliche Themen direkt und nicht abstrakt adressiert, wird dadurch möglich. Beispielhaft ist hier die abstrakte Industrie 4.0 Kompetenz des Gesteigerten Prozessverständnisses zu nennen, dessen Entwicklung nur durch Kenntnisse 
über den Prozess, die darin tätigen Menschen und kontextuelle Rahmenbedingungen zielführend organisiert werden kann. Im Ergebnis führt die Förderung von Individuen zu einer besseren fachlichen Passbarkeit von Angebot und Bedürfnissen, die ihrerseits einen hohen Zielerreichungsgrad in der Kompetenzentwicklung nach sich zieht. Ebenfalls kann der (digital modifizierte) Arbeitsprozess, auf den diese Kompetenzentwicklung abzielt, in der Realität dadurch besser gemeistert werden.

Paradox 3 Mitarbeitende werden zwar als Teil der digitalen Transformation gesehen, eine aktive Beteiligung am Wandel bleibt jedoch aus

Die Untersuchungsergebnisse zeigen, dass Mitarbeiter über die Bedeutung und Auswirkung der digitalen Veränderungen informiert werden: Die Vermittlung zentraler Veränderungen wird als ausreichend eingestuft. Gleichzeitig bestehen aus Sicht der Beschäftigten Kompatibilitätsprobleme zwischen neuen und alten Prozessen bzw. Technologien. Bei Letzteren liegt zudem oft ein mangelnder Reifegrad vor. Daran anschließend wurde oft eine fehlende bzw. geringe Beteiligung der Beschäftigten am Diskurs und der Umsetzung des digitalen Wandels deutlich. Paradox ist, dass Beschäftigte genau jenes Prozess- und Technikwissen mitbringen, dass für die Überwindung dieser Kompatibilitätsprobleme gebraucht wird, diese aber nicht aktiv in den Wandel einbezogen werden.

Wird betriebliche Weiterbildung als PD realisiert, profitieren nicht nur die daran Teilnehmenden. Durch den engen Austausch von Organisatoren und Nachfragern, der Gegenüberstellung von Angebot und Nachfrage, dem gegenseitigen Darlegen von Potenzialen und Risiken - kurz, der dialogorientierten Schaffung adäquater Weiterbildungsangebote - ist ebenfalls eine weiterführende Unterstützung des digitalen Wandels möglich. Einerseits bekommen technische Planer und Umsetzer sowie betriebswirtschaftlich handelnde Leitungspersonen die Möglichkeit, Bedürfnisse direkt bei z. B. von Kompatibilitätsproblemen betroffenen Personen abzuschöpfen. Darauf basierend können gemeinsam Lösungswege kreiert werden, die sowohl das praktische Problemverständnis der Mitarbeiter als auch die Problemlösekompetenz übergeordneter Führungskräfte verbinden. Gleichzeitig wird eine Kommunikation auf Augenhöhe ermöglicht, deren Zweck in der kollaborativen Problembewältigung liegt. Daraus resultiert eine höhere Akzeptanz der Beschäftigten gegenüber dem digitalen Wandel, da die digitale Transformation dadurch den Charakter eines ,Topdown" oktroyierten, abstrakten Prozesses verliert und eher zu einem realen Vorgang wird, den die Mitarbeiter aktiv beeinflussen können.

\section{Fazit}

Das Thema Weiterbildung ist als Kernanliegen im betrieblichen Alltag angekommen. Die Ausgestaltung von Weiterbildungs- und Qualifizierungsangeboten und -strategien bestimmt die Frage, ob Unternehmen Beschäftigte adäquat auf den digitalen Wandel vorbereiten können. Letzterer bestimmt als Zielgröße wiederum die notwendigen Mitarbeiterkompetenzen und demzufolge auch neue Anforderungen, die an Weiterbildung gestellt werden. 
Daran anknüpfend standen Auswirkungen der Digitalisierung auf die betriebliche Weiterbildung aus der Sicht der Mitarbeiter im Fokus der hier vorgestellten empirischen Untersuchung. Diese dienen als Grundlage, um Herausforderungen und Ansätze zu diskutieren, wie eine der wichtigsten Dienstleistungen im organisationalen Kontext etwas näher an die Person und weiter weg von der Masse gestaltet werden kann. Ausgangsgrößen hierzu sind relevante Eigenschaften, die als Grundlage für die individualisierte Kompetenzentwicklung dienen, sowie die Voraussetzungen, die individuelle Beteiligung an der Gestaltung des Wandels erlauben. Am Beispiel von drei Paradoxen wurde die Rolle verdeutlicht, die die Gestaltung betrieblicher Weiterbildung als PD spielen kann, um Unzulänglichkeiten bei der Verknüpfung von digitalem Wandel und Kompetenzentwicklung zu beseitigen.

Nachfolgende Untersuchungen sind vor diesem Hintergrund auf die Frage zu fokussieren, inwieweit und wie Unternehmen unterstützt werden können, um operativ und strategisch hinsichtlich Digitalisierungsprozessen die richtigen Entscheidungen zu treffen und ihre Beschäftigten passend auf die neuen Handlungskontexte vorzubereiten. Hierzu werden individualisierte (unter anderem mit wachsender Relevanz digitale) Weiterbildungsangebote, Mitarbeiterbeteiligung und Akzeptanz vermehrt eine Rolle spielen und die persönlichen Eigenschaften der Beschäftigten immer mehr in den Mittelpunkt rücken.

Förderung Diese Arbeit wurde durch das Bundesministerium für Bildung und Forschung (BMBF) gefördert (Förderkennzeichen: 16DII116 - „Deutsches Internet-Institut“).

Funding Open Access funding provided by Projekt DEAL.

Open Access Dieser Artikel wird unter der Creative Commons Namensnennung 4.0 International Lizenz veröffentlicht, welche die Nutzung, Vervielfältigung, Bearbeitung, Verbreitung und Wiedergabe in jeglichem Medium und Format erlaubt, sofern Sie den/die ursprünglichen Autor(en) und die Quelle ordnungsgemäß nennen, einen Link zur Creative Commons Lizenz beifügen und angeben, ob Änderungen vorgenommen wurden.

Die in diesem Artikel enthaltenen Bilder und sonstiges Drittmaterial unterliegen ebenfalls der genannten Creative Commons Lizenz, sofern sich aus der Abbildungslegende nichts anderes ergibt. Sofern das betreffende Material nicht unter der genannten Creative Commons Lizenz steht und die betreffende Handlung nicht nach gesetzlichen Vorschriften erlaubt ist, ist für die oben aufgeführten Weiterverwendungen des Materials die Einwilligung des jeweiligen Rechteinhabers einzuholen.

Weitere Details zur Lizenz entnehmen Sie bitte der Lizenzinformation auf http://creativecommons.org/ licenses/by/4.0/deed.de.

\section{Literatur}

acatech (2016) Kompetenzen für Industrie 4.0. Qualifizierungsbedarfe und Lösungsansätze. Herbert Utz, München

Acemoglu D, David A (2011) Skills, tasks and technologies: Implications for employment and earnings. In: Ashenfelter O, Card D (Hrsg) Handbook of labor economics, Bd. 4. Elsevier, Amsterdam, S $1043-1171$

Battelle for Kids (2019) Framework for 21st century learning definitions. http://static.battelleforkids.org/ documents/p21/P21_Framework_DefinitionsBFK.pdf. Zugegriffen: 17. Jan. 2020

Brynjolfsson E, McAfee A (2016) The second machine age: Work, progress, and prosperity in a time of brilliant technologies. W. W. Norton \& Company, New York, London 
Calvani A, Fini A, Ranieri M (2009) Assessing digital competence in secondary education. Issues, models and instruments. In: Leaning M (Hrsg) Issues in information and media literacy: Education, practice and pedagogy. Informing Science, Santa Rosa, S 153-172

Dengler K, Matthes B (2015) Folgen der Digitalisierung für die Arbeitswelt. Substituierbarkeitspotenziale von Berufen in Deutschland (IAB-Forschungsbericht 11/2015)

Hermann T, Hirschle S, Kowol D, Rapp J, Resch U, Rothmann J (2017) Auswirkungen von Industrie 4.0 auf das Anforderungsprofil der Arbeitnehmer und die Folgen im Rahmen der Aus- und Weiterbildung. In: Andelfinger V, Hänisch T (Hrsg) Industrie 4.0. Wie cyber-physische Systeme die Arbeitswelt verändern. Gabler, Wiesbaden, S 239-253

Hirsch-Kreinsen H (2014) Welche Auswirkungen hat Industrie 4.0 auf die Arbeitswelt? WISO direkt. Friedrich-Ebert-Stiftung, Bonn, S 1-4

Kagermann H, Wahlster W, Helbig J (2013) Promotorengruppe Kommunikation der Forschungsunion Wirtschaft und Wissenschaft: Umsatzempfehlungen für das Zukunftsprojekt Industrie 4.0. Stifterverband für die Deutsche Wissenschaft, Berlin

Klenow PJ (1998) Learning curves and the cyclical behavior of manufacturing industries. Rev Econ Dyn 1(2):531-550

Paproth Y, Kittel A (2019) Digitalisierung von individuellen Lernpfaden: Mitarbeiterbezogene und bedarfsgerechte Lernpfade für kleine und mittlere Unternehmen in der Industrie 4.0. UdZ-Forschung 2:22-24

Rai A, Patnayakuni R (1996) A structural model for CASE adoption behavior. J Manag Inf Syst 13(2):205-234

Ramos MAW, Figueiredo PS, Pereira-Guizzo C (2018) Antecedents of innovation in industry: The impact of work environment factors on creative performance. Innov Manag Rev 15(3):269-285

Russwurm S (2015) Software Die Zukunft der Industrie. In: Sendler U (Hrsg) Industrie 4.0: Beherrschung der industriellen Komplexität mit SysML. Springer Vieweg, Wiesbaden, S 21-36

Shapiro C, Carl S, Varian HR (1998) Information rules: a strategic guide to the network economy. Harvard Business Press, Harvard

Vladova G, Ullrich A, Reiff-Stephan J (2017) Der Mensch im Industrie 4.0 Umfeld. In: Weinert N, Plank M, Ullrich A (Hrsg) Metamorphose zur intelligenten und vernetzten Fabrik: Ergebnisse des Verbundforschungsprojekts MetamoFAB. Springer Vieweg, Wiesbaden, S 23-30

Zeit online (2017) Schluss mit der Euphorie. http://www.zeit.de/2017/18/digitalisierung-politik-bedrohungdemokratie-industrie-4-0-datenschutz/seite-3. Zugegriffen: 17. Jan. 2020

Gergana Vladova Dr., ist Postdoktorandin am Lehrstuhl für Wirtschaftsinformatik, insb. Prozesse und Systeme an der Universität Potsdam und Leiterin der Forschungsgruppe „Bildung und Weiterbildung“ in der digitalen Gesellschaft am Weizenbaum Institut für die Vernetzte Gesellschaft in Berlin. Seit 2008 forscht und lehrt sie zu den Themen Bildung und Weiterbildung, Kompetenzentwicklung im Kontext der Digitalisierung, Wissens- und Innovationsmanagement und Produktpiraterie.

Alexander Heuts arbeitet als wissenschaftlicher Mitarbeiter am Weizenbaum-Institut für die vernetzte Gesellschaft in der Forschungsgruppe „Bildung und Weiterbildung in der digitalen Gesellschaft“ in Berlin und am Lehrstuhl „Didaktik der Informatik/Informatik und Gesellschaft“ an der Humboldt-Universität zu Berlin.

Malte Teichmann arbeitet als wissenschaftlicher Mitarbeiter am Lehrstuhl für Wirtschaftsinformatik, insb. Prozesse und Systeme an der Universität Potsdam und am Weizenbaum-Institut für die vernetzte Gesellschaft, Potsdam und Berlin, Deutschland. 